ORIGINAL RESEARCH PAPER

\section{A PROSPECTIVE STUDY OF FUNCTIONAL AND RADIOLOGICAL OUTCOIME OF PERTROCHANTERIC FEMUR FRACTURES TREATED WITH PROXIMAL FEMORAL NAIL ANTI ROTATION II ( PFN A2)}

Orthopaedics

KEY WORDS:PFN A2

Intertrochanteric fracture, modified harris hip score

\section{Dr Kannelli}

Maneesh

Chandra*

\section{Dr A Sandeep}

Kumar
Post Graduate, Department Of Orthopaedics, Narayana Medical College And Hospital-524003, Nellore, Andhra Pradesh, India. *Corresponding Author

Senior Resident , Department of Orthopaedics , Narayana Medical College And Hospital -524003, Nellore , Andhra Pradesh ,India

Post Graduate , Department of Orthopaedics , Narayana Medical College And Hospital-524003, Nellore, Andhra Pradesh ,India

Dr Mudra Dinesh

BACKGROUND: Femoral Inter-Trochanteric fracture is one of the most frequently occurring fractures in the elderly, usually following trivial trauma. In the younger age group of people, it occurs almost always due to high velocity trauma. OBJECTIVES: This study aimed to study the functional and radiological outcomes of operative management of intertrochanteric fracture treated by PFN-A2.

MATERIAL \& METHODS: A prospective study of 20 cases of intertrochanteric femur fracture treated by PFN-A2, minimum 6 months of follow up. All cases are evaluated according to Modified Harris Hip Score on residual effects on clinical ground at final examination. Pain \& functional capacity are the two basic considerations for this scoring system. Points are given for pain, function, range of motion \& absence of deformity.

RESULT: Functional result according to Modified Harris Hip score was found to be excellent in $11(55 \%)$ patients, good in $5(25 \%)$ patients, fair in $3(15 \%)$ patients \& poor in $1(5 \%)$ patients. poor outcome occurs due to development of complications.

CONCLUSION : PFN A2 has the advantage of minimal incision, shorter operative time, rapid rehabilitation, lower medical complications among other options.

\section{INTRODUCTION}

Intertrochanteric fracture is the fracture of the proximal femur, in which the fracture line extends from the greater trochanter to the lesser trochanter. These fractures are common in patients in the geriatric age group, due to the increase in the prevalence of osteoporosis. However, it may also occur in young adults due to high-velocity trauma. Nine out of ten intertrochanteric fractures occur in the elderly, the female to male ratio is $3: 1^{1}$.

These fractures are usually classified as Stable and Unstable Fractures. The characteristics of an unstable variety are posteromedial fragmentation, basicervical patterns, reverse obliquity patterns, displaced greater trochanteric (lateral wall communition) fractures ${ }^{2}$.

Conservative management of these fractures is fraught with complications of prolonged recumbency as well as limp and shortening due to malunion in coxa-vara ${ }^{3}$. Extramedullary implants such as DHS, once considered the solution to these fractures have performed less than satisfactorily in unstable patterns paving way for intramedullary implants $\mathrm{s}^{4-6}$. but in unstable intertrochanteric fractures, it has a higher incidence of cut-out failure $(6 \% \text { to } 19 \%)^{7,8}$. However, an intramedullary device with a shorter lever is likely to improve the biomechanics providing more load sharing and limiting collapse at the fracture site ${ }^{9}$.

The proximal femoral nail anti-rotation (PFNA), a modification of proximal femoral nail (PFN), was introduced in 2003, which features a helical blade. Biomechanical cadaveric studies demonstrated that PFNA fixation using a helical blade was better compared to the sliding hip screw. PFNA, characterized by rotational along with angular stability has biomechanically improved purchase in the osteoporotic bone due to the bony impaction it achieves in the femoral head and neck ${ }^{10,11}$. PFNA was designed for femoral geometric proportions of the Caucasian population but differences exist between Asian and Caucasian femoral geometry ${ }^{12}$. Serious complications occurred when PFNA was used for Asians ${ }^{13}$ which led AO/ASIF to design a new proximal femoral nail anti-rotation Asia (PFNA
2) for Asian femoral geometry ${ }^{14}$.This study was undertaken to analyze the results of unstable intertrochanteric fracture of femur fixed with PFNA 2, its functional and radiological outcomes in the elderly Indian population.

\section{MATERIALS AND METHODS :}

A prospective study was carried out from October 2019 and September 2020 in the Department of Orthopaedics, Narayana medical college, and hospital, Nellore. A total of 20 patients with unstable intertrochanteric fractures were treated surgically by proximal femoral nail - A2 and were followed up at the immediate post-op period and Postoperative radiological outcome was assessed by periodic $\mathrm{X}$ rays of affected hip and evaluated for clinic-functional outcome using Modified Harris Hip Score.

\section{INCLUSION CRITERIA :}

1. Patients $>18$ years of age presenting to our causality with intertrochanteric femoral fractures with Boyd and Griffin types 2-4.

2. Fractures less than 1-week duration

3. Without any other associated fractures

\section{EXCLUSION CRITERIA:}

1. Fractures with non-union changes

2. Old malunited intertrochanteric fracture

3. Patients with arthritic changes in hip and knee joint

4. Pathological fractures

5. fractures over ipsilateral lower limb and patients with neck of femur and shaft of femur fractures

After getting Ethics committee clearance and informed \& written consent from the patients and attenders, the patients enrolled in the study were subjected to thorough clinical examination. The baseline investigations and radiographic analysis such as plain X-ray of the affected hip with proximal femur were analyzed. All the cases were posted for surgical management with closed or open reduction with PFN-A2 and followed up according to our study protocol

Patients were initially managed with intravenous fluids, whole |www.worldwidejournals.com $\mid$ 
blood transfusion (if needed) \& then limb rested in upper tibial pin traction or skin traction to immobilize and maintain the length \& alignment of the fractures.

All cases were given with IV antibiotics for 5 days followed by oral antibiotics for 5 days. The active and passive exercises were initiated within 48 hours of surgery. All patients with osteoporosis were treated with vitamin D, calcium supplements, bisphosphonates, and teriparatide in severe cases.

For Type II fractures with stable fixation patient allowed weight bearing as tolerated from day 2 to 10. Full weight bearing on the adequate union in follow-up x-rays. For unstable fracture patterns weight-bearing delayed and partial weight-bearing continued for 4 to 6 weeks with touch toe walking and full weight-bearing in the following weeks as per the follow-up x-rays. All patients were followed up at the end of 1, 2, 6, and 12 months. At each follow-up, the radiographs of the upper femur and hip were taken to assess the fracture union and the complications. The functional results were calculated according to the Modified Harris hip score
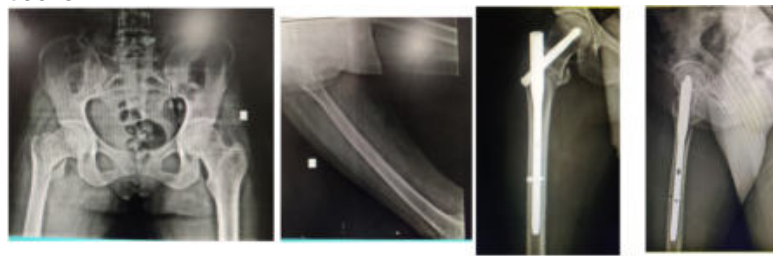

Fig 1. a, preop ap view b, preop lateral view c, post op ap view $d$, post lateral view Case 1 showing pre op and post op $x-$ rays
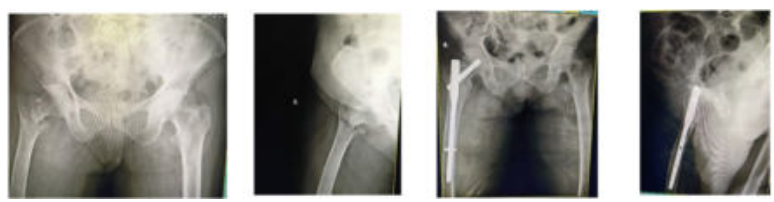

Fig 2 . a, preop ap view b, preop lateral view c, post op ap view d, post lateral view

\section{Case 2 showing pre op and post op $x$-rays}

\section{RESULTS}

All cases were followed up for a minimum of 12 months and were assessed for clinical, radiological, and functional outcomes. The results were analyzed. The observations of our study are as follows:

The age groups varied from 52 years to 75 years with a mean age of 60 years. There was a female preponderance with 13 females and 7 males, suggesting osteoporosis is higher among female population. Fall due to slip and fall was the most common mode of injury. 12 patients suffered a fracture on the right side and 8 patients suffered a fracture on the left side. Boyd and Griffin type II (unstable) fractures are the most common type in our study followed by type IV and type III.

The time duration of surgery of the patients varied from 37 mins to 98 mins with a mean of 46 mins Majority of patients were operated on within 7 days, the average being 4.6 days. Mean blood loss was $220 \mathrm{ml}$. The mean length of the incision was $5 \mathrm{~cm}$. The mean helical blade size was $85 \mathrm{~mm}$.

The average hospital stay was 7 days. Partial weight-bearing in most cases was allowed immediately on the $3 \mathrm{rd}$ postoperative day based on construct stability and bone quality. All fractures united on an average of 12.4 weeks. All patients were allowed to full weight bearing on an average by 12 weeks based on the clinical and radiological union.

|www.worldwidejournals.com
According to Modified Harris Hip scores, out of 20 cases, the functional outcome was excellent in 11 cases (55\%), good in 5 cases $(25 \%)$, fair in 3 cases (15\%), and poor in 1 case $(5 \%)$. The poor range of movements $(n=1)$ was due to varus collapse and no helical blade cut-out is seen.

Table 1 , modified harris hip scores at final follow up

\begin{tabular}{|l|l|l|}
\hline Modified Harris hip score & No. of cases & Percentage \\
\hline Excellent 81-100 & 11 & $55 \%$ \\
\hline Good 61-80 & 5 & $25 \%$ \\
\hline Fair 41-60 & 3 & $15 \%$ \\
\hline Poor < 40 & 1 & $5 \%$ \\
\hline
\end{tabular}

\section{COMPLICATIONS}

One female patient a known case of Type 2 diabetes mellitus suffered from wound infection and got healed after wound wash and 3 weeks of antibiotic. One patient had varus collapse. One patient had persistent hip pain Weight-bearing was deferred in this patient and pain was relieved after the radiological union.

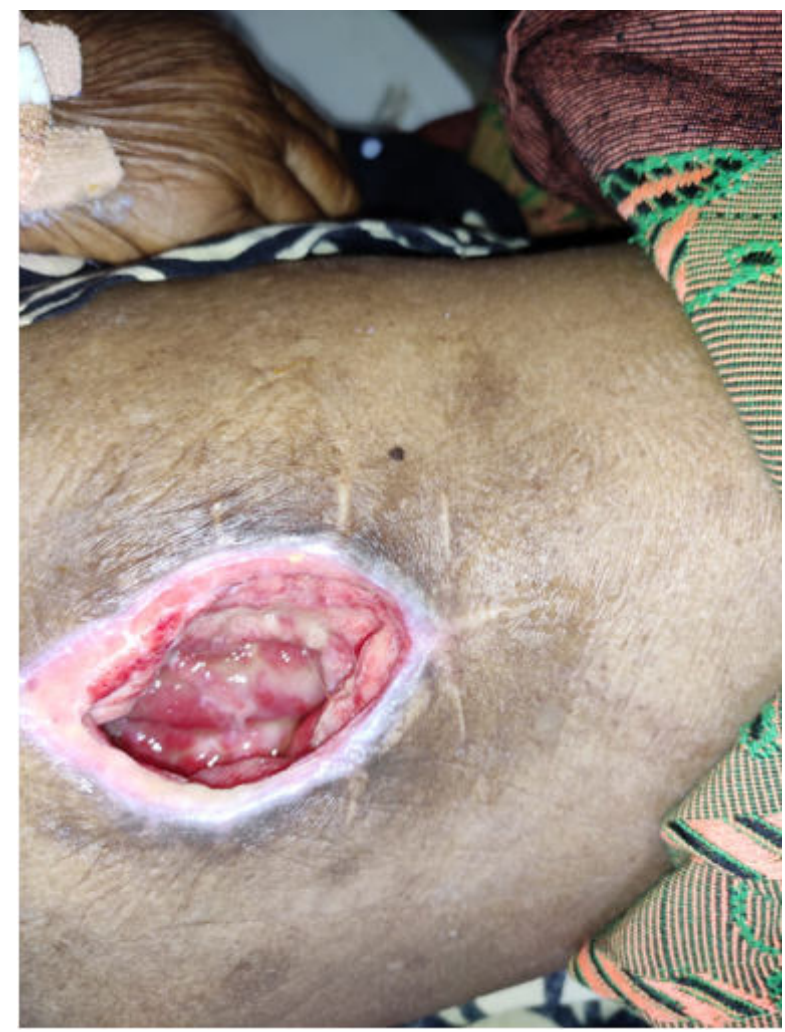

Figure 3 - wound infection at surgical site

\section{DISCUSSION}

The management of unstable intertrochanteric fractures poses a significant challenge to orthopedic surgeons. These fractures represent a technical difficulty in reduction of fractures and implication of surgical implant may lead to failure of the primary fixation of a fracture. The medial and posteromedial fracture fragments were the most important elements in determining the severity of intertrochanteric fracture $^{15}$. The recent implant for management of unstable intertrochanteric fracture was proximal femoral nail antirotation-2. PFN-A2 has advantages over PFNA in the following ways:

a) the proximal nail diameter was reduced from $17 \mathrm{~mm}$ to $16.5 \mathrm{~mm}$

b) the mediolateral angle was reduced from 6 degrees to 5 degrees, and

c) a flat proximal lateral surface was adapted to avoid impingement of femoral lateral cortex ${ }^{16,17}$. In this modern era, researchers stated that the important predictor for reoperation after the trochanteric fracture is the lateral femoral wall ${ }^{18}$. 
Bhatti et al. ${ }^{19}$ concluded Proximal Femoral Nail was associated with reduced blood loss, shorter hospital stays less morbidity compared with Dynamic Hip Screw. Klinger et al. ${ }^{20}$ did a comparative study of unstable intertrochanteric fractures and concluded that Proximal Femoral Nail was associated with shorter operation time, shorter hospital stays, higher rate of patients with early full weight-bearing, fewer complications compared with Dynamic Hip screw Simmermacher et al. ${ }^{21}$

concluded that PFNA currently is an optimal implant concerning the prevention of femoral head penetrations for the treatment of unstable trochanteric fractures. E. Soucanye de Landevoisin et al. ${ }^{22}$ concluded that PFNA has additional benefits in patients with osteoporotic trochanteric fractures, both by preventing rotation and by ensuring cancellous bone compaction. This design may diminish the rate of complications associated with the cervical implant, provided the implantation procedure is scrupulously followed and fracture reduction is optimal.

Macheras et al. $^{23}$ concluded that PFNA II avoided lateral cortex impingement experienced with PFNA, providing fast and stable fixation of the unstable peritrochanteric fractures. Gururagavendra et al. ${ }^{24}$ concluded that both the implants (PFN and PFN-A2) have comparable radiological and functional outcomes for unstable intertrochanteric fracture except for less surgical time and blood loss in PFN A2. The superiority of one implant over another could be arrived at with still larger sample size, involving multi-center studies.

In our study, a new cephalo-medullary proximal femoral nail anti-rotation-2 (PFN-A2) for unstable intertrochanteric fractures was used. These nails prevent the rotation and collapse of the head-neck fragment and the smaller diameter of the distal shaft of the nail results in less stress concentration at the tip of the nail. The anti-rotation screw at the proximal aspect of the nail increases the biomechanical stability of the fracture fixation.

Limitations of the study were smaller sample size; limited duration of post-operative follow up to comment on the biomechanical stability of the implant and no control group for comparison

\section{CONCLUSION}

Unstable intertrochanteric fractures pose a great challenge for orthopedic surgeons to manage surgically. Due to the evolution of load-sharing devices, it is possible to manage the fractures with internal splinting. We conclude that the proximal femoral nail anti-rotation 2 (PFN-A2) was an ideal implant for unstable intertrochanteric fractures, leading to a high rate of bone union restoring the lateral femoral wall, reducing the chances of implant failure, and decreasing the post-operative morbidity by improving the functional quality of life with following benefits

1. Smaller Incision,

2. Less Bood Loss \&Shorter Operative Time Due To Usage Of A Single Helical Blade,

3. Significant Cut Out Resistance Due To Impacted Helical Blade,

4. Shorter Individuals With Short Neck Can Accommodate A Single Screw Rather Than Two In Other Devices.

5. Rapid rehabilitation

\section{REFERENCES}

1. Frederick MA, Terry SC, James HB. Campbell's operative orthopaedics in treatment of intertrochanteric fractures (13th edn),Vol.3, p. 2828 .

2. Sun-Jun H (2016) PENA-2 protrusion over the greater trochanter in the Asian population used in proximal femoral fractures. Ind J of Orthop 50(6):641-646. Falch JA, Liebekk A, Slungaard U. Epidemiology of hip fractures in Norway. Acta Orthop Scand. 1985;56(1): 12-16.doi:10.3109/17453678508992970

3. Falch JA, Liebekk A, Slungaard U. Epidemiology of hip fractures in Norway. Acta Orthop Scand. 1985;56(1): 12-16. doi: 10.3109/17453678508992970

4. Russel TA. Intertrochanteric fractures of the hip. In: Court-Brown CM, Heckman JD, McQueen MM, Ricci WM, Tornetta III Paul, McKee MD, editors. Rockwood and Green's fractures in adults. 8th ed. Philadelphia: Wolters Kluwer Health;2015.p 2075-129.

5. Gundle R, Gargan MF, Simpson AH. How to minimize failure of fixation of 140 unstable intertrochanteric fractures. Injury. 1995; 26(9): 611-4. doi: 10.1016/0020-1383(95)00125-s.

6. Simpson $A H$, Varty $\mathrm{K}$, Dodd $\mathrm{CA}$. Sliding hip screws: modes of failure. Injury. 1989;20(4):227-31. doi: 10.1016/0020-1383(89)90120-4

7. Domingo L, Cecilia D, Herrera A, Resines C: Trochanteric fractures treated with a proximal femoral nail. Int Orthop. 2001, 25:298-301. $10.1007 / \mathrm{s} 002640100275$

8. Harrington P, Nihal A, Singhania AK, Howell FR: Intramedullary hip screw versus sliding hip screw for unstable intertrochanteric femoral fractures in the elderly.Injury. 2002,33:23-28.10.1016/s0020- 1383(01)00106-1

9. Adams CI, Robinson CM, McQueen MM: Prospective randomized controlled trial of an intramedullary nail versus dynamic screw and plate for intertrochanteric fractures of the femur. J Orthop Trauma. 2001, 15:394- 400. 10.1097/00005131-200108000-00003

10. Liu Y, Tao R, Liu F, Wang Y, Zhou Z, Cao Y, Wang H: Mid-term outcomes after intramedullary fixation of peritrochanteric femoral fractures using the new proximal femoral nail antirotation (PFNA). Injury. 2010, 41:810-817. 10.1016/j.injury.2010.03.020

11. Strauss E, Frank J, Lee J, Kummer FJ, Tejwani N: Helical blade versus sliding hip screw for treatment of unstable intertrochanteric hip fractures: a biomechanical evaluation. Injury. 2006, 37:984-989. 10.1016/j.injury.2006.06.008

12. Siddiqi N,Valdevit $A$, Chao EY:Differences in femoral morphology among the Orientals and Caucasians: a comparative study using plain radiographs. Ana Sci Int. 2019,94:58-66. 10.1007/s 12565-018-0450-1

13. Li J, Cheng L, Jing J: The Asia proximal femoral nail antirotation versus the standard proximal femoral antirotation nail for unstable intertrochanteric fractures in elderly Chinese patients. Orthop Traumatol Surg Res. 2015, 101:143-146.10.1016/j.otsr.2014.12.011

14. Lv C, Fang Y, Liu L, Wang G, Yang T, Zhang H, Song Y: The new proximal femoral nail antirotation-Asia: early results. Orthopedics. 2011, 34:e18-e23. 10.3928/01477447-20110317-26

15. Pajarinen J, Lindahl J, Michelsson O, Savolainen V, Hirvensalo E (2005) Pertrochanteric femoral fractures treated with a dynamic hip screw or a proximal femoral nail. A randomised study comparing post-operative rehabilitation.J Bone Joint Surg Br 87(1): 76-81.

16. Boblee J, Ram P, Vijayakumaran (2017) Functional outcome of proximal femoral nailing in intertrochanteric fractures of femur: A prospective study. Int J Orth Sci 3(2):513-518.

17. Harshwardhan H, Jain S, Sharma M (2019) An outcome analysis of intertrochanteric fracture of femur managed with proximal femoral nail antirotation II. International Journal of Research in Orthopaedics 5(4):699.

18. Gotfried Y (2004) The lateral trochanteric wall: a key element in the reconstruction of unstable pertrochanteric hip fractures. Clin Orthop Relat Res 425:82-86.

19. Bhatti A, Power D, Qureshi S, Khan I, Tan S (2004) A prospective trial of proximal femoral nail vs. dynamic hip screw for unstable intertrochanteric fractures of the femur.J Bone Joint Surg Br 86B(3): 377

20. Klinger HM, Baums MH, Eckert M,Neugebaur R (2005) A comparative study of unstable per and intertrochanteric femoral fractures treated with Dynamic Hip Screws and trochanteric buttress plate vs. Proximal Femoral Nail Zentralbl Chir 130(4):301-306.

21. Simmermacher RKJ (2008) The new proximal femoral nail antirotation (PFNA) in daily practice: Results of a multicentre clinical study Injury. Int J Care Injured 39(8): 932-939.

22. Soucanye de LE (2012) Proximal femoral nail antirotation (PFNATM) fixation of extra-capsular proximal femoral fractures in the elderly: Retrospective study in 102 patients. Orthopaedics \& Traumatology: Surgery \& Research 98: 288-295.

23. Macheras (2012) Does PFNA II avoid lateral cortex impingement for unstable peritrochanteric fractures? Clin Orthop Relat Res 470(11):3067-3076

24. Gururagavendra P, Sathish D, Jayakumar S, Devadoss A (2018) Randomised comparative study in management of unstable intertrochanteric fracture with PFN V/S PFN A2 - functional and radiological outcome. Int J Orthop Sci 4(4): 866-874. 